\title{
MODELING OF CONTINUOUS MELTING OF QUARTZ- FELDSPAR RAW MATERIALS OF SUBSEQUENT FLOW FROM THE PLASMA HEATING
}

\author{
Mariya S. Malashenko ${ }^{1}$, Eugene A. Maslov ${ }^{\mathrm{a}, 2}$, Boris V. Borisov ${ }^{1}$ \\ ${ }^{1}$ National Research Tomsk Polytechnic University, 634050 Tomsk, Russia \\ ${ }^{2}$ National Research Tomsk State University, SRIAMM, 634050 Tomsk, Russia
}

\begin{abstract}
The problem of obtaining easy-flowing melt silicate-containing materials with the use of low-temperature plasma. A mathematical formulation of the evolution of the silicate-containing raw material to the melt. The analysis of the preliminary results.
\end{abstract}

\section{INTRODUCTION}

Getting easy-flowing melt silicate-containing refractory materials is expensive energy. Sometimes, to solve this problem is proposed use of the energy of low-temperature plasma. Currently, this approach is becoming more common, in particular for producing high silicate melts under continuous feed into the crucible, and simultaneously removing from the latter finished melt [1,2]. Schematically, the structure for this technology (Fig. 1). As the analysis available in the literature experimental data and numerical simulation results [3]. The use of the plasma column is associated with significant heat fluxes, the parameters of which are related to the characteristics of the electrical conductivity of the feed raw material and melt, which essentially depends on the aggregate state, and temperature. Local source powder inlet silicate-containing materials having a relatively low temperature, leads to a rather abrupt temperature field nonuniformity that can affect both the performance of the overall process (e.g., sintering into larger conglomerates) and create thermal stresses in the structure of the crucible, which can lead to equipment failure.

Mathematical modeling of thermal processes in the considered design avoids unnecessary costs in establishing the most effective modes of technology acquisition and development of high-silicate melt from the waste of energy and mining industry using the energy of low-temperature plasma. In this case, the total cross-sectional area, free to move in this direction of the flow varies from a minimum at the entrance to the flow of raw materials up to a maximum at the intersection of the axis of the cylindrical crucible and again to a minimum at the expiration of the finished melt (Fig. 2).

In accordance with a change in the geometry of the flow cross section and the flow rate varies. The minimum radial velocity is in the axis of the crucible, where the stream is most intense thermal effect, which favorably affects the final result, in terms of the completeness of the greatest melt. On the other hand achieve a melt temperature higher than necessary, leading to thermal decomposition of the raw material required to loss of useful properties. Preliminary analysis of the modes of fusion by means of mathematical modeling, taking into account the maximum design features allowing for an early design

a Corresponding author: maslov_eugene@mail.ru 
stage to choose the most rational modes of loading of raw materials, melt and discharge parameters of the plasma column.

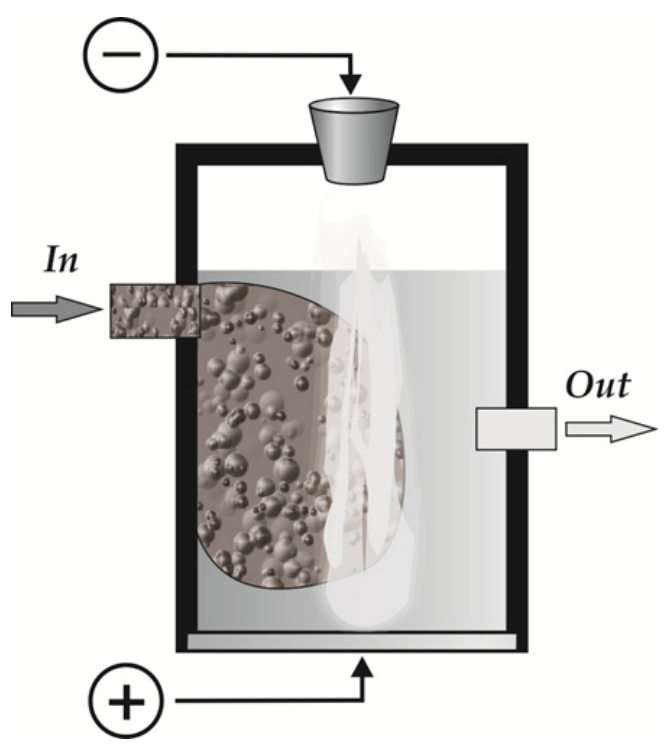

Figure 1. Scheme of experimental setup

\section{TECHNIQUE OF MATHEMATICAL MODELING}

In this paper we consider the problem of particles injecting the melting pot of raw materials to the further advancement of these particles over the area of the flat section of the crucible in a high viscous incompressible flow melt. Particles are injected into a high temperature at an initial flow rate and temperature. The temperature of the injected particles is lower than the temperature of the carrier flow, $T_{p}<T_{f}$.

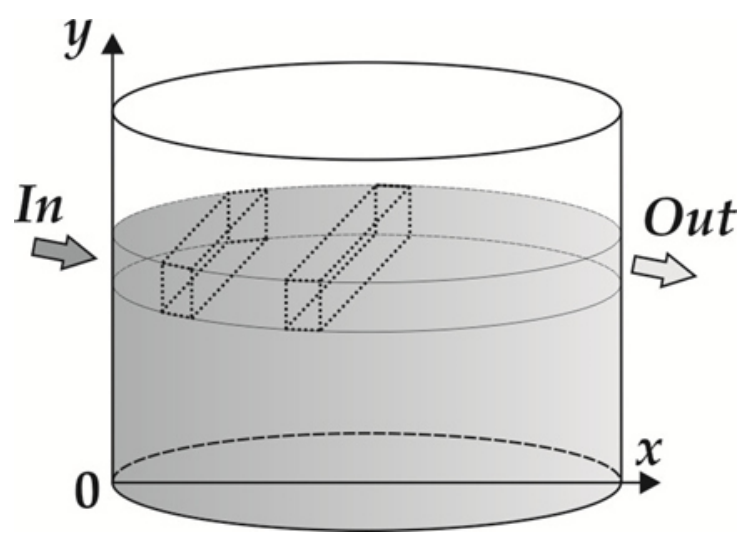

Figure 2. Scheme of region the solution.

Melting occurs in the stream when the condition $\mathrm{Tp}=\mathrm{Ts}-$ equality of the melting point temperature of the particles. Interaction of the flow with the wall surface is performed by Newton's law (molecular dynamic fluid friction of a smooth surface) [3]. The particles interact with the wall surface of the crucible in operation of sliding friction [4].

As the analysis of the calculation results [2]:

1. the flow is stationary and subsonic substantially laminar, 
2. viscous effects can be simulated completely shear stresses in the model of Newton,

3. the density of the raw material feed though varies according to the composition and porosity components sufficiently close to the density of the melt is determined and the same temperature dependence as the melt.

As part of the physical setting for the fluid is described by unsteady equations of motion of the Navier - Stokes equations with the variable area, free for the flow:

$$
\begin{aligned}
& \frac{\partial u S_{x}}{\partial t}+u \frac{\partial u S_{x}}{\partial x}+v \frac{\partial u S_{y}}{\partial y}=\frac{\partial}{\partial y}\left(v S_{y} \frac{\partial u}{\partial y}\right)-\frac{S_{x}}{\rho} \frac{\partial p}{\partial x} \\
& \frac{\partial v S_{y}}{\partial t}+u \frac{\partial v S_{x}}{\partial x}+v \frac{\partial v S_{y}}{\partial y}=\frac{\partial}{\partial x}\left(v S_{y} \frac{\partial v}{\partial x}\right)-\frac{S_{y}}{\rho} \frac{\partial p}{\partial y}-\beta g S_{y}
\end{aligned}
$$

Equations (1) and (2) are closed by the continuity equation:

$$
\frac{\partial u S_{x}}{\partial x}+\frac{\partial v S_{y}}{\partial y}=0
$$

The flux density is generally a function of temperature and volume fraction of unmelted feedstock:

$$
\rho=\rho\left(T(t, x, y), r_{p}\right),
$$

To determine the pressure field in the approach formulated in [4], after transformations (1) - (3) obtained by the Poisson equation:

$$
\left(\frac{\partial u S_{x}}{\partial x}\right)^{2}+\left(\frac{\partial v S_{x}}{\partial x}\right)^{2}+\frac{\partial v}{\partial x} \frac{\partial u S_{x}}{\partial y}+\frac{\partial v S_{y}}{\partial x} \frac{\partial u}{\partial y}=-\nabla^{2} p-g S_{y} \frac{\partial \beta}{\partial y}
$$

Evolution unmelted feedstock described by the equation for its volume fraction rp. Simulated moving at the speed of the environment, the local conductive heat flow $q_{k}$ and the local volumetric heat from the plasma column $q_{v}$ to the melting temperature $T_{s}$ and the subsequent melting at a constant temperature $T_{s}$ to extinction:

$$
\frac{\partial r_{p}}{\partial t}+\frac{\partial r_{p} u}{\partial x}+\frac{\partial r_{p} v}{\partial y}=-\frac{k_{F V} q_{k}+q_{v}}{q_{s} \rho_{p}}
$$

where $q_{s}, \rho_{p}$ - respectively, the specific heat of the phase transition (melting), and the average density of the starting material mixture, $k_{F V}$ - coefficient determining the ratio between the surface and the volume of the starting powder mixture.

Modelling of the thermal field of the crucible is carried out using the energy equation, which allows heat to expended to melt flow of the particles of the starting material at $r_{p}>0$ :

$$
\frac{\partial c_{p} T}{\partial t}+\frac{\partial c_{p} u T}{\partial x}+\frac{\partial c_{p} v T}{\partial y}=\frac{1}{\rho}\left[\frac{\partial}{\partial x}\left(\lambda \frac{\partial T}{\partial x}\right)+\frac{\partial}{\partial y}\left(\lambda \frac{\partial T}{\partial y}\right)+q_{v}-q_{s} \rho_{p} \frac{\partial r_{p}}{\partial t}-k_{w V} q_{w}\right]
$$

In this equation $k_{w V}$ - the ratio between the area of cooling and arbitrary volume $V$. Numerical simulation was carried out by setting on the wall of the crucible of constant density of heat loss $q_{w}$, simulating the process of water cooling of the crucible.

On the walls of the channel are defined terms "sticking". On the free surface conditions are set of free slip and adiabatic. In place of the feed given constant flow temperature $T_{i n}$, considered for irrotational, the longitudinal component of the velocity is constant over the cross section value $u_{i n}$, the transverse component of the velocity is zero. On the exit surface of the melt from the crucible set "soft" boundary conditions.

\section{Conclusion}

For the numerical implementation of the described mathematical formulation was applied scheme [5]. Comparative analysis of the results of preliminary calculations and the experimental data obtained show the physical adequacy of the presentation of mathematical models of the process. To calculate 
the chosen thermal parameters and transference the corresponding parameters of quartz glass. Shown substantial reduction in flow velocity in the radial region of the axis of the crucible as compared with [4]. There are the modes of heat loss $q \omega$, in which feed is partially sank to the bottom and moving along it completely melt reaches the point of emergence from the crucible. According to calculations by adjusting the water cooling process can achieve optimal parameters for which the raw material at the outlet of the crucible can be fully molten, and at the same time, the wall temperature does not exceed the permissible level.

The study was performed by a grant from the Russian Science Foundation, project №15-19-10014.

\section{References}

1. Volokitin O, Timonov E, Volokitin G, Nikiforov A, Chibirkov V. Pat. Rus. Fed. N 2503628. Plasma apparatus for synthesis of refractory silicate melt.

2. Volokitin O, Volokitin G, Shekhovtsov V. Physicochemical processes at plasma treatment of quartz-feldspar raw materials. Advanced Materials Research. 2015 V. 1085 pp. 474-478.Maslov, at al., Gas-dynamic functions, (Moscow, Mashinostroenie, 1965)

3. Volokitin O, Maslov E., Borisov B., Shekhovtsov V. Modeling of continuous melting of quartzfeldspar raw materials with use the plasma technology. 2014 pp. 545-548 (NPNJ2014, may 2531, Alushta, Russia)

4. Roache, P.J. 1976, Computational Fluid Dynamics (Albuquerque: Hermosa).

5. Patancar, S. 1980, Numerical heat transfer and fluid flow (New York, Hemisbhere Pu,lishing Corporation) 